\title{
Application of color displays to problems in the undergraduate laboratory
}

\author{
DOUGLAS R. EDDY \\ Trinity University, San Antonio, Texas 78284
}

\begin{abstract}
Some topics in psychology are difficult to master without dynamic visual displays. This paper describes a few applications of computer-driven color graphics displays in an undergraduate laboratory in neuropsychology. The paper includes the advantages, disadvantages, and cost of this approach, as well as student reactions and evaluations.
\end{abstract}

Durrett (1979) discusses the different types of color video displays available; this paper attempts to partially answer the following question: What can you do with color that is useful? Although the primary purpose of this paper is to describe the application of a particular set of color display terminals to a specific set of undergraduate laboratory problems, several other questions of a more general nature will be addressed. The particular applications implemented in our undergraduate laboratory stemmed from our goal to achieve educational objectives that would be difficult or impossible without a computer. Our implementations, then, revolve around simulations and on-line analysis of real-time psychological data. The use of simulations in teaching concepts is not a new application of computers, but the use of computers for analysis of analog physiological data in the undergraduate laboratory is. In fact, in 1977, the CONDUIT Psychology Committee (Note 1) did not include a category for on-line real-time computer uses in their classificatory scheme for instructional packages employing computers.

Since the color video terminals are only a part of the system, I will briefly describe the current system, the current applications, and some related and more general questions.

\section{COMPUTER SYSTEM AND COLOR TERMINALS}

A Texas Instruments 960 process control computer is currently the computational workhorse for all programs discussed in this paper. The system has $32 \mathrm{~K}$ 16-bit words of memory, two 1.1-million-word disk drives, hardware arithmetic, an eight-channel A/D converter, two interval timers, two digital cassettes,

Requests for reprints, information, and source program listings should be addressed to Douglas R. Eddy, Department of Psychology, Trinity University, 715 Stadium Drive, San Antonio, Texas 78284. Programming support and the Intecolor terminals were supported in part through cost-sharing RULE and ISEP grants from the National Science Foundation (SER76-14507 and SER77-10916). The author gratefully acknowledges the programming efforts of Mary Beth Rosson, Penny Lee Smith, Lex Bakarich, and Daniel Laser. a hard-copy printer, and six 9,600-baud terminals. The system is connected to four laboratories through a central switching circuit allowing the A/D channels to switch from one laboratory to the other.

The computer's operating system, the process automation monitor (PAM/D), is disk resident. PAM/D handles all task scheduling when multiple tasks are running simultaneously. The system has a FORTRAN II-based compiler called PCL (for process control language) that contained many enhancements to FORTRAN for process control applications. This compiler is supplemented by a preprocessor that translates structured FORTRAN statements (Friedman \& Koffman, 1977) into FORTRAN II, allowing multiple-alternative decision structures and the While loop structure. The system has an assembler, three editors, and other utilities, such as a linkage editor for linking subroutines and creating overlays and a librarian task for cataloging various tasks and subroutines. Central to our computing philosophy is PAM/D's ability to generate reentrant routines, allowing several students to interact with the same program simultaneously but independently. The system has other embellishments in both hardware and software, but these are not used in the current curriculum implementations.

The six 8001 Intecolor terminals were purchased from Intelligent Systems, Inc., in kit form. These terminals were assembled by undergraduate engineering students and debugged by some of our computer center personnel. Even though this was one of the best documented kits we had ever assembled, I do not recommend purchasing such a complicated electronics system in kit form. The manufacturer probably agrees, since the kit is no longer available from Intelligent Systems.

These terminals have the following specifications: 19-in. color CRT, ASCII character set, eight colors, 48 80-character lines, $8080 \mathrm{CPU}, \mathrm{RS} 232 \mathrm{I} / 0$ ports, and graphics software with a screen resolution of 160 by 192 points. The software, stored in programmable read-only memory (PROM), has a command language allowing foreground and background color, visible and blind cursor positioning, page and roll modes, selectable baud 
rates, several plot modes including point plot, vector plot, and incremental plot, horizontal and vertical histograms, and erase (points, lines, and page). These terminals are rolled on carts to the various laboratories where they are used for instructional and research purposes. Connections are made to the minicomputer through hard wires from each of the laboratories on three different floors of the building. Standard EIA interface cards are used to communicate at 9,600 baud with the computer. Originally, it was hoped that these terminals could be easily modified to connect with the campus computer, an IBM 370/155. Since these terminals did not have an APL character set, work on connecting the units had to await the efforts described by Rodgers (1979).

\section{APPLICATIONS}

The programs discussed were inserted into the freshman laboratories of a course entitled, "The Biological Bases of Behavior." Each laboratory contains approximately 25 students and is concerned with the neuroanatomy of the cow brain, sensory systems (especially the ear and skin), and some demonstration experiments, usually on modification of EEG by biofeedback or photic driving.

The first laboratory involves the demonstration of sound waves and a discussion of how they stimulate the ear. Students are exposed to sound waves aurally over a speaker and visually on an oscilloscope screen. Both single and multiple frequencies are used. The computer is used to show the students how pure sine waves coming from more than one oscillator can be combined into a complex signal or wave. Each group of four students works with a terminal, keying in the necessary information used to generate sine waves of varying amplitudes and frequencies. The students also indicate, after prompting, the colors in which they would like each wave plotted. After entering the parameters for each wave, the computer plots the wave across the terminal's screen. After entering parameters for up to nine waves, the students may combine any or all waves to see what the composite complex wave looks like. Additional options include plotting the generated wave on a colored background and causing the plotted wave to blink. Since resolution of the screen is limited, only frequencies between 1 and $10 \mathrm{~Hz}$ and amplitudes between 1 and $10 \mathrm{~V}$ are allowed. Through this interactive demonstration, students can conceptualize more complex speech sounds that are later shown on an oscilloscope. The laboratory session next moves to a discussion of how the auditory mechanisms decode complex sounds in to simple sine-wave components through a process similar to Fourier analysis.

This simple reentrant program allows the student to see how complex waves can be made from simple sine waves without going through all the mathematics-skills college freshmen have often not acquired. The original program for performing all of these functions was written by an undergraduate student in psychology. Most students in the laboratory participate enthusiastically. Of course, a few are enthusiastic for the wrong reasons. With different background colors and blinking, some students attempt to generate images that are artistically pleasing rather than trying to learn something about complex waves. However, from questions asked by the students, both during the laboratory session and later in studying the ear and how it decodes sound waves, it is obvious that they understand the relationships demonstrated in the program. The color graphics make it possible to show many different waves without confusing them, which would be very difficult on a two-color screen. While we have no experimental control groups, we feel that color is an important motivator for student participation.

A second freshman laboratory exercise is an analysis technique used in research laboratories in neuropsychology, namely, percentage power spectral analysis of EEG (brain waves). To introduce this procedure into the undergraduate laboratory, we standardly use the biofeedback paradigm to show how a human can voluntarily control his own EEG. The EEG is displayed on an oscilloscope so that the students can observe it, and the subject is instructed to use a deep relaxation technique that causes changes in the dominant brain rhythms. The EEG is spectrally analyzed at various times during the session and displayed graphically on the terminal screen showing the percentage power contribution of each frequency component in the EEG sample. Different colors are used to plot the analysis for each electrode and thus make it easy for the students to see differences between the two hemispheres.

A third laboratory demonstration relies heavily on the terminal's capability for selective erasure of a portion of the screen without affecting other parts of the screen. Silberstein, Nastronarde, and George (1976) programmed the Hodgkin-Huxley equations for predicting all of the aspects of a nerve impulse in the giant fibers of the squid and the Dodge (1963) and Hille (1971) equations for the fibers in the frog sciatic nerve. These equations were adapted for our system, displaying the results graphically in color. The display uses eight lines of color-coded alphanumeric information at the top of the screen and two graphs at the bottom. Each graph plots two of eight possible output variables, membrane voltage, its first derivative, sodium current, potasium current, all ionic currents summed, and the parameters of the equations $\mathrm{N}, \mathrm{H}$, and $\mathrm{M}$. One graph plots the two selected variables over time as the neuron fires; the other plots successive pictures of the two variables as they would be seen if a short segment of the simulated nerve fiber could be viewed. In the latter graph, the two lines plotted for the variables are erased and then replotted with the new values at the next 
time increment. Since the partial differential equations are solved for each time increment after stimulation, the student can see activity taking place inside a nerve fiber during the action potential.

This type of visual display is virtually indispensable for understanding the ionic and electrical relationships within the neuron. Now that our Intecolor terminals are compatible with the IBM 370 (Rodgers, 1979), we are hoping to run a simplified version of this simulation on the campus computer. This would allow students to run the program interactively through several different kinds of neuron experiments, including voltage clamp, twin pulse stimulation, and threshold determination.

A fourth laboratory project involving color-coded sections of the human brain is in the beginning stages of implementation. First, a section of the brain is drawn on graph paper. Then, each line segment and its color are entered into a disk file. The program, which is only about 50 source statements, decodes the information as it reads it from the disk and draws a picture of that section. The student can then ask for labels or request the program to test him by drawing a line to one of the structures and waiting for a response. This program has two main objectives: It allows the student to associate well defined parts of the brain with a certain name, and it teaches him through drill how to spell the name correctly (e.g., amygdala). Final testing of the student's understanding of neuroanatomy is always carried out with real brain specimens. It is hoped that the colorcoded displays provide a smoother transition from the textbook to the real thing. This project was initiated by a graduate student as a final project in a class entitled, "Computers in the Behavioral Sciences."

A fifth project involves simulating some of the simple neural networks using lateral inhibition for detecting slits, lines, edges, and movement taken from Lindsay and Norman (1977). We are designing a simple command language that would allow students to build simple neural networks to account for the relationships between simple patterns of light on the retina and the responses of cells in the visual cortex. This project will take advantage of the new software described by Rodger (1979), designed to simulate the movement of light across a bank of retinal receptor cells. The receptor cells excite ganglion cells that are inhibited by adjacent receptor cells acting through horizontal cells. Changing the connections, gains, and delays in the network allows the students to create different types of detectors. These are then tested with various light patterns, comparing the quantitative output of the network with what would be required for a specific kind of detector.

\section{STUDENT FEEDBACK}

Questionnaire responses at the end of the semester on approximately 48 students revealed that $42.2 \%$ felt that the computer system had contributed to their understanding of the course subject matter, $35.6 \%$ felt that it had not, and $22.2 \%$ were neutral. Other response items on the questionnaire asked if the laboratory was informative and if it was interesting. Although there were strong correlations between these questions and the question of the computer's contribution, indicated by significant chi-square tests, it was interesting to compare the cells of particular rows and columns. Of 16 students who felt that the laboratory was informative, 9 felt that the computer displays had contributed, 6 felt that they had not, and 4 were neutral. Of those who found the laboratory interesting, 12 felt that the computer had contributed to their understanding of the material, 2 did not, and 3 were neutral. Since the numbers in these cells are so small, I could not accept these results as reliable, but I think they are indicative that the computer courseware and the color terminals add most noticeably to interest in the course. The question is still unsettled as to whether the computer courseware makes a substantial contribution to the students' understanding of the course content.

\section{CONTRIBUTION OF COLOR DISPLAYS}

A big advantage of multicolor displays over two-color displays is that important information in a display can be made to stand out. Colored line graphs are much easier and more natural for the eye to follow; color is more direct. In displaying text, key words can be accentuated, helping a reader to organize the material more easily. Color is a more engaging medium than twocolor displays-something that is easier to experience than to talk about.

A disadvantage of color displays is that they take more programming effort. They also require more planning time in creating the displays to take advantage of the color. After the novelty wears off, color displays may prove distracting, but I doubt it.

The Intecolor terminals have some limitations. The 8001 terminal receives and processes each character it receives from a host computer. This means that the next character cannot be sent until the last one is processed or it will be lost. Processing time varies for each command and even within some commands. For example, in drawing a line from point " $A$ " to point " $B$," longer lines require more time. This has created problems for use since we would like to send information as fast as possible for some displays, not as slow as the worst case.

In the Intecolor 8001 , color is controlled by character. This means that even though there are eight plottable points within a character, each point must be either the foreground color or the background color. Therefore, within a character there can be no more than two colors. When two lines of a different color cross on a third background color, the points in the first line are changed to the color of the second. The resolution of the color on the screen is in reality only 48 by 80 .

Under our version of the Intecolor graphics software, 
if a point is erased from the screen twice, as when two lines cross and both are to be erased, the point of crossing is turned back on by the second erasure. After several erasures, the screen can become cluttered with little dots. The standard ASCII backspace, control " $\mathrm{H}$," homes the cursor on the 8001. This is a real problem when correcting input from the keyboard that is being sent to the host computer. Finally, when a color terminal is physically moved or rotated, the color guns must be reconverged for a clear, perfect picture. This can be easily accomplished in $5 \mathrm{~min}$ with Intecolor's convergence system.

There appear to be some weaknesses in the 8001 hardware, specifically in the high-voltage section of the analog board. This problem may have been corrected in a later system, however. Some of our terminals also frequently blow fuses after they are moved. Since we assembled the terminals ourselves, it is not possible to tell yet whether these are design or assembly problems.

The configuration in which we bought our terminals requires that they be connected to a host computer. If you wish to do the same, make sure that the host computer can handle the number of terminals and the baud rate.

In writing software for color graphics systems like the 8001 , use structured programming techniques, modularize everything, take some time developing efficient algorithms, and use higher level languages whenever possible.

\section{$\operatorname{CosT}$}

The Intecolor 8001 terminal kits with the options mentioned earlier cost approximately $\$ 2,000$ each. We paid two engineering students $\$ 800$ to assemble the kits. The power-up sequence required the services of a technician familiar with digital logic. Our technicians spent nearly $1,000 \mathrm{~h}$ looking for solder bridges, replacing bad components, making adjustments, and so on, before all six terminals were up and running. If these individuals had not been willing to take on these responsibilities and we had hired someone to do this work, it would have been far less expensive for us to purchase the terminals in assembled form. An assembled system (as of November 1978) costs $\$ 2,750$. Assembled hardware should be purchased whenever possible.

\section{REFERENCE NOTE}

1. CONDUIT Psychology Committee. Computers in teaching psychology-A state of the art report. Iowa City, Iowa: CONDUIT, 1977.

\section{REFERENCES}

DoDge, F. A. A study of ionic permeability changes in mylinated nerve fibers in the frog. Unpublished doctoral dissertation, Rockefeller University, 1963.

DurRetr, H. J. Color display systems: The state of the art. Behavior Research Methods \& Instrumentation, 1979, 11, 127-130.

Friedman, F. L., \& Koffman, E. B. Problem solving and structured programming in FORTRAN. Reading, Mass: AddisonWesley, 1977.

HiLle, B. Voltage clamp studies of mylinated nerve fibers. In W. J. Adelman (Ed.), Biophysics and physiology of excitable membranes. New York: Van Nostrand, 1971.

LindSAY, P. H., \& NoRMAN, D. A. Human information processing: An introduction to psychology (2nd ed.). New York: Academic Press, 1977.

RODGERS, F. Microprocessor software applications in color graphics. Behavior Research Methods \& Instrumentation, 1979, 11, 135-138.

Silberstein, P. T., Nastronarde, D. N., \& George, S. A. Teaching nerve impulses by computer in a neurobiology course. In T. C. Willoughby (Ed.), Proceedings of 1976 conference on computers in the undergraduate curricula, 1976 Binghamton Proceedings. New York: Computer Center, State University of New York at Binghamton, 1976. 